\title{
Specialization and semantic organization: Evidence for multiple semantics linked to sensory modalities
}

\author{
J. FREDERICO MARQUES \\ University of Lisbon, Lisbon, Portugal
}

\begin{abstract}
The present article reviews the case for multiple systems in semantic memory and empirically evaluates a multiple semantics proposal based on sensory modalities. In the experiments, a conceptual switching cost paradigm was used (Pecher, Zeelenberg, \& Barsalou, 2003), in which participants verified properties for concepts and verification time was compared for target trials (e.g., a $d o g$ can bark) that were preceded by context trials of either the same or a different modality (e.g., a bee can buzz or a horse can have spots). Experiment 1 involved a modality switch while controlling for the concept's category and demonstrated that when modalities were switched, a processing cost was incurred that could not be attributed to the latter dimension. Experiment 2 further supported this conclusion by showing that, in a reverse situation, the cost incurred when category was switched was at least smaller. The results are discussed by considering possible alternative amodal explanations and other data that have shown the influence of sensory information in conceptual processing.
\end{abstract}

The organization of concepts in semantic memory has been a central topic in the field of cognitive psychology for several decades (see, e.g., Chang, 1986, for a review). Since the early 1980s, this topic has received even wider attention stemming from research in cognitive neuropsychology. In fact, a huge number of diverse cases of brain-lesioned patients who have been held to have specific domain or category ${ }^{1}$ impairments involving the semantic system have been reported (for reviews, see, e.g., Capitani, Laiacona, Mahon, \& Caramazza, 2003; Forde \& Humphreys, 1999). In order to explain these cases and, ultimately, normal functioning, very different theoretical models of semantic memory organization have been proposed (for reviews, see, e.g., Caramazza \& Mahon, 2003; Forde \& Humphreys, 1999). One of the major discussions between models is whether the semantic system is better conceptualized as a unitary amodal store or as composed of multiple stores that are more or less independent and have been linked to different sensory modalities (including sensorimotor information) and/or domains of knowledge. Regarding this discussion, three positions can be distinguished that correspond to the three main current

This research was supported by the Centro de Psicometria e Psicologia da Educação da Universidade de Lisboa. I thank Larry Barsalou, Diane Pecher, and Jules Davidoff for their helpful discussions of and comments on earlier versions of the manuscript. I also thank Bill and Grace Bingham for their thorough proofreading. Finally, I thank Ana Sofia Morais, Inês A. Pinto, Joana Carmo, and M. Francisca Fonseca for their help in data collection. Correspondence should be addressed to J. F. Marques, Faculdade de Psicologia e de Ciências da Educação, Universidade de Lisboa, Alameda da Universidade, 1649-013 Lisbon, Portugal (e-mail: jfredmarq@fpce.ul.pt). theories of semantic organization: the modality-specific theory, the conceptual structure theory, and the domainspecific theory.

The modality-specific theory offers an explanation of cases of impairment that maintains that they reflect an underlying organization of specific modality-processing channels. The idea of multiple semantic stores linked with different sensory modalities can be traced more systematically to the work of Warrington and associates (e.g., Crutch \& Warrington, 2003; McCarthy \& Warrington, 1988). Building initially from the verbal-visual distinction, ${ }^{2}$ Warrington and associates proposed a multiple processing channels view of semantic memory. The salience of the different sensory and motor channels for the acquisition of knowledge would explain the organization of meaning (McCarthy \& Warrington, 1988; see also Shallice, 1988), and fine-grained categorical dissociations would arise as a consequence of different weighting values between the information from each of the channels. A second view, developed by Shallice and associates (e.g., Lauro-Grotto, Piccini, \& Shallice, 1997; Shallice, 1988, 1993), proposes a highly interconnected multimodal network. In this distributed network, the primary form of specialization was defined in terms of the different types of semantic operation that would occur predominantly with the respective complementary types of input (Lauro-Grotto et al., 1997; Shallice, 1988, 1993).

The conceptual structure theory offers an explanation of cases of impairment by assuming random damage to a unitary amodal system in which the correlation between features (especially the correlation between perceptual and function features) and their degree of distinctiveness govern the organization of information (e.g., Moss, 
Tyler, \& Devlin, 2002; Tyler, Moss, Durrant-Peatfield, \& Levy, 2000). This theory proposes that domains differ in terms of their correlated structures and their shared versus distinctive features, with living things possessing more shared features and correlated shared features and objects possessing more distinctive features and correlated distinctive features. This difference, associated with the assumption that features that are highly correlated with other features are more resistant to brain damage, would especially explain the disproportionate number of cases in which the domain of living things seems impaired, the converse deficit occurring only when damage to the semantic system is severe (e.g., Moss et al., 2002; Tyler et al., 2000).

Finally, the domain-specific theory offers a straightforward explanation of cases of impairment that states that they reflect an underlying organization in terms of domains of knowledge (Caramazza \& Mahon, 2003; Caramazza $\&$ Shelton, 1998). This theory proposes a direct organization in terms of evolutionarily salient object domains (especially animals, plant life, and tools) that would have been established on the basis of survival and reproductive advantages (Caramazza \& Mahon, 2003; Caramazza \& Shelton, 1998). In its first formulation (Caramazza \& Shelton, 1998), the preference was to link the theory with a unitary amodal account of semantic memory, the organized unitary content hypothesis (Caramazza, Hillis, Rapp, \& Romani, 1990), which maintained that items with similar properties would be stored in adjacent neural areas. More recently, Caramazza and Mahon (2003) have pointed out that the domain-specific theory is also commensurable with modality specificity (this possibility was only alluded to in Caramazza \& Shelton, 1998). Available evidence would be consistent with an organization of conceptual knowledge by modality-specific constraints within domain-specific processing channels or with the reverse (Caramazza \& Mahon, 2003). The former possibility is, however, explicitly preferred considering that the first-order constraint on the organization of conceptual knowledge is object domain (Caramazza \& Mahon, 2003).

Since the proposals for multiple semantics and, also, for unitary semantics are very general, it is extremely difficult to differentiate between them with regard to cases of impairment. In fact, some cases could be readily interpreted in terms of both of the alternative proposals as reflecting either a subsystem impairment or problems in accessing a full representation (and also with respect to the contribution of presemantic processing; for discussions, see, e.g., Caramazza \& Shelton, 1998; Shallice, 1993).

Evidence from cognitive psychology and behavioral data has been used to evaluate the question mainly in terms of the verbal-visual distinction and is not relevant to the present debate. Recently, however, Pecher, Zeelenberg, and Barsalou (2003) have provided evidence with normal subjects in favor of a specialization in terms of sensory modalities. They showed that verifying features associated with different modalities in successive trials (e.g., a target trial of blender-loud preceded by context trial of cranberries-tart) produced a switching cost in relation to successive same-modality trials (e.g., a target trial of blender-loud preceded by a context trial of leaves-rustling). In fact, verification time was longer for the former case, which is similar to the switching costs produced by detection signals from different modalities at the perceptual level (Spence, Nicholls, \& Driver, 2001). Moreover, this result could not be attributed to other factors, such as associative priming between features of the same modality (Pecher et al., 2003, Experiment 2), expectancy effects (successive same- and different-modality trials presented in mixed blocks and with a high ratio of filler trials), or any presemantic factors, since only words were used and all of the features were verbal, although related to different modalities (i.e., vision, motor action, audition, taste, touch, and smell). This switching cost was thus conceptual and, as such, provided a more decisive argument for multiple semantics. Pecher et al. concluded that conceptual processing is grounded in different sensory systems and that the cost results from processing switches from one modality system to another.

However, this study did not manipulate or control for the concept's domain and category, and thus, it may be disputed whether modality makes an independent contribution to the results. In fact, by considering the parallel with perceptual switching costs, it is also possible to find at this level situations that reflect processing costs of changing from one type of stimulus to another within the same presentation modality (e.g., Kavcic, Krar, \& Doty, 1999; Los, 1999). It is thus possible that the fact that trials also differed in terms of domain/category may have contributed to the switching costs. Moreover, if the domain-specific theory is taken into consideration, it is important to ensure that there is no possible confusion between modality and domain effects.

The main purpose of the present study was to disentangle these two possible effects in Pecher et al.'s (2003) experiments, evaluating their respective contributions within a modality-specific versus a domain-specific theory. Replicating the experiment while controlling for domain (and category) also makes it possible, using also the results in Pecher et al., to complete a possible parallel between these normal switching costs at the semantic level and those associated with cases of impairment described at the modality level (i.e., the different aphasias) and more specifically confined at the domain level within a certain modality. Finally, a replication of the experiment in which domain (animals vs. nonliving) is changed while sensory modality information is controlled for can also complement this evaluation and make possible a further test of the influence of the conceptual domain on property verification, following the same switching cost principle.

These questions were addressed in two experiments: Experiment 1, in which a modality switch was evaluated while concept domain was controlled for, and Experiment 2 , in which a concept domain switch was evaluated while feature modality was controlled for. ${ }^{3}$ 


\section{EXPERIMENT 1}

This first experiment replicated Pecher et al.'s (2003) study while controlling for concept domain, thus allowing an evaluation of the possibility of modality-switching costs in a situation in which successive trials were composed of items from the same conceptual domain-either both animals or both nonliving things. ${ }^{4}$

To ensure that feature selection and manipulation reflected modalities in terms of the salience of the latter to feature acquisition, we previously had had a larger set of features rated in terms of how audition, vision, and touch contributed to their acquisition and knowledge. This is an important evaluation, since some features can be acquired by or, at least, simultaneously activate different modalities (e.g., a horse's mane can activate/be acquired by vision and touch). This multimodal nature of stimulus evaluation has been extensively demonstrated at the perceptual level in terms of crossmodal links in both spatial attention (e.g., Spence, Pavani, \& Driver, 2000) and tactile perception (e.g., Sathian \& Zangaladze, 2002). At the level of semantic organization and in relation to category-specific dissociations, this aspect has been considered less. Authors either have contemplated the overall perceptual nature of features and emphasized visual versus verbal semantics (e.g., Farah \& McClelland, 1991; Warrington \& Shallice, 1984) or have considered the contribution of different perceptual modalities independently (Cree \& McRae, 2003; McCarthy \& Warrington, 1988).

On the basis of the general switching costs demonstrated by Pecher et al. (2003) and taking into consideration the evidence in favor of a multiple semantics in terms of sensory modalities, it was expected that in this situation, in which the conceptual domain was constant, modality-switching costs would also be observed in successive different-modality trials, in comparison with the same-modality trials.

\section{Method}

Participants. Sixty-two undergraduate students from the University of Lisbon participated for partial fulfillment of an introductory psychology course requirement. Twenty-five participated in the preliminary feature-rating task, and 37 participated in the main experiment. From the latter group, 1 participant was eliminated due to technical problems with the response box during the experiment. All analyses of the main experiment were based on a total sample of 36 participants.

Materials. Ninety-five concept plus feature pairs (e.g., a rocket can be large) were considered for the rating task (all the materials were in Portuguese). In an adaptation of Tranel, Logan, Frank, and Damasio's (1997) procedure, the participants were asked to rate, on a 5-point scale, the degree to which audition, vision, and touch contributed to the knowledge of that concept's feature (i.e., large for rocket). Each participant rated the 95 pairs separately for the three modalities, with modality order counterbalanced between participants. The criterion by which a feature was considered to have a dominant modality of knowledge was that it should have a mean rating higher than 4 for that modality and, simultaneously, that it should have a mean rating lower than 3 for the remaining two modalities. Eighty-two items from the total sample satisfied this criterion and were used to build up the 64 critical target and context trials (see the Appendix). The remaining items and the nondominant items (which mainly indicated a similar dominance for touch and vision) were used for the filler trials.

Thirty-two concept-feature pairs were selected for the target trials (i.e., the trials that followed a trial of the same or a different feature modality), of which half consisted of animals and another half of nonliving things (arms, clothes, furniture, and tools). Thirty-two concept-feature pairs were then selected for the context trials (i.e., the trials that preceded a target trial of the same or a different feature modality), which consisted of concepts of the same domain and category as those of the target trials but which presented either a feature of the same modality ( 8 with animals and 8 with nonliving things) or a feature of a different modality $(8$ with animals and 8 with nonliving things). Two lists were created in such a way that each target trial had a same-modality context trial in one list but a different-modality context trial in the other, as is shown in Table 1.

Thus, each target trial appeared with both same-modality and different-modality context trials, counterbalanced across lists (all the features were true of their respective concepts). These 64 critical trials were also controlled for in terms of concept familiarity between animals and nonliving things (Portuguese familiarity norms from Marques, 1997) and, more important, were controlled for in terms of association, so that neither the concepts nor the features were associated between consecutive context and target trials (Portuguese free association norms from Marques, 2002). In addition to the 64 critical trials, the experiment included 124 filler trials, designed to mask the nature of the experiment. Within the filler trials, 92 were false (i.e., the feature was not true of the concept), and 32 were true (i.e., the feature was true of the concept). The filler trials were arranged in pairs (like the context + target trials) as follows: 30 false + false trial pairs, 16 false + true pairs, and 16 true + false pairs, consisting, in each case, half of animal + animal items and half of nonliving things + nonliving things items. Thus, true and false responses were almost equally likely overall ( $49 \%$ false and $51 \%$ true items). The features in the fillers referred to a dominant modality or to multiple modalities. As in Pecher et al. (2003), to ensure that the participants actually verified the features, the concept and feature in many of the false items were semantically related (e.g., squid-fish, sofa-rest). The critical (i.e., context + target trials) and the filler pairs were randomly intermixed for each participant. Also, the concepts and features were used only once. The practice trials were similar in nature to the experimental trials, consisting of 12 true items and 12 false items. Materials were arranged in two separate blocks by conceptual domain (animals and nonliving things) and were mounted on computer-presented slides, using the SuperLab for Windows software.

Procedure. Each trial began with a 500-msec fixation stimulus $(* * * * *)$, which was followed by a concept-feature pair that was framed in a sentence "a CONCEPT can be/have FEATURE." The sentence remained on the screen until the participant responded by pressing a two-button response box, with the preferred hand being assigned to the yes button and the other to the no button (the right hand was preferred by $95 \%$ of the participants). The initial instructions emphasized that a yes/no decision should be based on whether the feature was "usually true" of the concept and that the participant should try to respond as quickly as he or she could. The participants received feedback for $600 \mathrm{msec}$ after pressing the wrong key ("ERROR") or after taking 2,000 msec or longer to respond ("TOO

Table 1

Examples of the Target and Context Trials in Experiment 1

\begin{tabular}{lll}
\hline & \multicolumn{2}{c}{ Context Trial } \\
\cline { 2 - 3 } Target Trial & Same Modality & Different Modality \\
\hline Dog-bark & Bee-buzz & Horse-pinto \\
Worm-flaccid & Lobster-rough & Deer-brown \\
Telephone-ring & Clock-tick-tock & Mirror-reflect \\
Lamp-light & TV-colors & Mattress-damp \\
\hline
\end{tabular}


SLOW"). An intertrial blank slide appeared for $500 \mathrm{msec}$ after the participant had responded correctly or after the feedback when the response was wrong or too slow.

The experiment began with the 24 practice trials, followed by one block of trials, a small resting period (about $1 \mathrm{~min}$ ), and the second block of trials. Block order (i.e., blocks of animals and nonliving things) was counterbalanced between participants.

As in Pecher et al. (2003), because the participants responded to each trial individually and because only $17 \%$ of the trial transitions involved features from the same modality, there was no indication that there was any kind of pairing between trials or that modality switching was of interest. This was also confirmed, in the present study, by questioning the participants after the experiment, with no participant claiming to have noticed anything particular about the trials or about the trial transitions (only that one block had been composed of animals and another of different objects).

\section{Results and Discussion}

Reaction times (RTs) were considered only for target trials with a correct response on both the target trial and the preceding context trial, given that an assessment of modality switching assumes that the participants processed both the context and the target items correctly. RTs exceeding 2,000 $\mathrm{msec}$ (the too slow limit) were eliminated from the analyses (outliers corresponded to $2 \%$ of the responses) but were not counted as errors. Median RTs and percentages of errors for the same modality versus the different modality on target trials were calculated for each participant and then were averaged for the total group $(N=36)$. These results are presented in Table 2 by modality switch condition (switch vs. no switch) and target item category (animals vs. nonliving things).

The results were analyzed by participant with a $2 \times 2$ repeated measures ANOVA with switch condition and domain as factors, ${ }^{5}$ and showed that RTs on the target trials were slower when the modality switched from the context trial to the target trial than when the modality remained constant $\left[F(1,35)=5.03, M S_{\mathrm{e}}=9,248, p<.05\right]$. No other main effects or interactions for RTs (although RT switching costs seemed to be higher for animals) or errors were significant [although errors were a little higher for same-modality trials, the difference was not significant$F(1,35)=2.23, M S_{\mathrm{e}}=172.02, p<.14$-and thus, there was no speed-accuracy trade-off]. These data support a modality-specific theory, maintaining that conceptual processing is grounded in different sensory systems and that the cost results from processing switches from one modality to another. Moreover, these data confirm the data obtained by Pecher et al. (2003) and extend their conclu- sions to a situation in which processing switches can be attributed only to modality. These results are also consistent with a domain-specific theory in which modality-specific systems are organized by first-order domain-specific constraints (Caramazza \& Mahon, 2003). This interpretation was further evaluated in Experiment 2.

\section{EXPERIMENT 2}

In this experiment, the possibility of a conceptual domain switch when modality is kept constant was examined. As was previously mentioned, the results from Experiment 1 are consistent with an organization by firstorder domain-specific processing channels and with an organization by modality within those channels. From this hypothesis, it follows that we should also expect conceptual domain switches when modality is kept constant, since these would correspond to a switch between major domain-specific processing channels. If this domain organization corresponds to a specialization that is orthogonal to modality and constitutes a first-order constraint, we should expect even larger switch costs in this situation, which is the reverse of that in Experiment 1.

\section{Method}

Participants. Thirty-eight undergraduate students from the University of Lisbon participated for partial fulfillment of an introductory psychology course requirement. Two participants were eliminated for presenting more than $25 \%$ errors in the target trials. All the analyses were based on a total sample of 36 participants.

Materials. The same database as that established for Experiment 1 was used to build the critical context and target trials. Twenty concept-feature pairs were selected for the target trials (i.e., the trials following a trial of the same or a different feature modality), of which half consisted of animals and another half of nonliving things (arms, clothes, furniture, and tools). Twenty concept-feature pairs were then selected for the context trials (i.e., the trials that preceded the target trial of the same or a different feature modality), which consisted of items with a feature of the same modality but which were of either the same category or a different category from that of the target (i.e., animals vs. nonliving things). As in Experiment 1, two lists were created in such a way that each target trial had a samecategory context trial in one list but a different-category context trial in the other, as is shown in Table 3.

Thus, each target item appeared with both same-category and different-category contexts, counterbalanced across lists (all the features were true of their respective items). These 40 critical trials were also controlled for in terms of familiarity between animals and artifacts (Portuguese familiarity norms from Marques, 1997) and, more important, were controlled for in terms of association, so

Table 2

Mean Reaction Times (RTs, in Milliseconds) and Error Rates (in Percentages) of Attribute Verification on Target Trials by Context Trial and Conceptual Domain (With Standard Errors)

\begin{tabular}{|c|c|c|c|c|c|c|c|c|}
\hline \multirow[b]{3}{*}{ Context Trial } & \multicolumn{4}{|c|}{ Animals } & \multicolumn{4}{|c|}{ Nonliving Things } \\
\hline & \multicolumn{2}{|c|}{ RT } & \multicolumn{2}{|c|}{ Errors } & \multicolumn{2}{|c|}{ RT } & \multicolumn{2}{|c|}{ Errors } \\
\hline & $M$ & $S E$ & $M$ & $S E$ & $M$ & $S E$ & $M$ & $S E$ \\
\hline Same modality & $1,083.97$ & 30.70 & 17.31 & 2.26 & $1,107.06$ & 26.10 & 17.63 & 2.22 \\
\hline Different modality & $1,125.18$ & 28.73 & 13.44 & 2.29 & $1,137.72$ & 24.41 & 14.97 & 2.65 \\
\hline Switching cost & -41.21 & & 3.87 & & -30.66 & & 2.66 & \\
\hline
\end{tabular}


Table 3

Examples of the Target and Context Trials in Experiment 2

\begin{tabular}{lll}
\hline & \multicolumn{2}{c}{ Context Trial } \\
\cline { 2 - 3 } Target Trial & \multicolumn{1}{c}{ Same Category } & Different Category \\
\hline Dog-bark & Lion-roar & TV-low \\
Telephone-ring & Clock-tick-tock & Donkey-heehaw \\
Worm-flaccid & Octopus-slippery & Sweater-cottony \\
Skirt-rough & Scarf-smooth & Bear-furry \\
\hline
\end{tabular}

that between consecutive context and target trials, neither items nor features were associated (Portuguese free association norms from Marques, 2002). In addition to the 40 critical trials, the experiment included 80 filler trials, designed to mask the nature of the experiment. Within the filler trials, there were 60 filler trials in which the feature was not true of the item and 20 trials in which the feature was true of the item. The filler trials were arranged in pairs (like the context + target trials) as follows: 20 false + false trial pairs, 10 false + true pairs, and 10 true + false pairs, consisting, in each case, half of animal + animal items and half of artifact + artifact items. Thus, true and false responses were equally likely overall. The features in the fillers referred to a dominant modality or to multiple modalities and also included many false related items. The critical (i.e., context + target trials) and filler pairs were randomly intermixed for each participant. Also, the items and features were used only once. The practice trials were the same as those in Experiment 1. All the materials were mounted in a single block of trials on computerpresented slides, using the SuperLab for Windows software.

Procedure. The procedure was the same as that in Experiment 1 with respect to general instructions, slide sequence on each decision, answer mode, and recording.

\section{Results and Discussion}

RTs were considered only for target trials with a correct response on both the target trial and the preceding context trial. RTs exceeding 2,000 msec (the too slow limit) were eliminated from the analyses (outliers corresponded to $3 \%$ of the responses) but were not counted as errors. Median RTs and percentages of errors for the same-category versus the different-category trials on target trials were then calculated separately by item category (living and nonliving) for each participant and then averaged for the total group $(N=36)$. The results are presented in Table 4 by category switch condition (switch vs. no switch) and target item category (animals vs. nonliving things).

The results were analyzed by participant with a $2 \times 2$ repeated measures ANOVA with switch condition and item category as factors, but no significant effects were found for RTs or errors. For the category switch crucial dimension, switch cost differences are in the "right" direction but are not significant $\left[F(1,35)=0.10, M S_{\mathrm{e}}=18,748\right.$, $p<.74$ for RTs, and $F(1,35)=1.33, M S_{\mathrm{e}}=209.21, p<$ .25 , for errors]. Taking into consideration from Experiment 1 that a comparable effect size of .40 could be expected according to Cohen's (1988) terminology, a power analysis showed that power was .92 (for $n=36$ and $p=$ $.05)$, which is clearly above the conventional .80 specified by Cohen (1988) to detect an effect. However, if a medium size effect was to be posited, power would be reduced to .39 , which clearly is below Cohen's recommendation.

In summary, the results from Experiment 2 do not support an organization of semantic memory by domain as a first-order constraint. Although conceptual cost switches by domain were observed, they were neither significant nor larger than the modality switches in Experiment 1. With regard to the contribution of the conceptual domain to property verification, all that can be said from the present results is that any possible effect of this dimension seems to be smaller than the one related to modalityassociated feature acquisition. As such, the results are not inconsistent with an organization by domain-specific subsystems within modality-specific processing channels, a possibility admitted by Caramazza and Mahon (2003). This possibility will be further analyzed in the General Discussion section.

\section{GENERAL DISCUSSION}

In the present article, the evidence for multiple systems of semantic memory has been analyzed in terms of the role of sensory modalities in the organization of semantic memory, and this proposal has been evaluated using behavioral data obtained with normal participants. This evaluation was done with a feature verification task and a switching cost framework considered at the conceptual level. The first experiment replicated Pecher et al.'s (2003) results for modality switch costs in a situation in which the concept's domain (and also the category) was kept constant. The second experiment gave additional support to this interpretation in terms of modality. In fact, by failing to show a comparable category switch cost effect in a reverse situation (i.e., a category switch, with modality kept constant), the results clearly separate the contribution of sensory modality from a possible smaller contribution of the conceptual domain to conceptual processing in this situation. Overall, the results are more in accordance with a view of semantic memory organization that proposes

Table 4

Mean Reaction Times (RTs, in Milliseconds) and Error Rates (in Percentages) of Attribute Verification on Target Trials by Context Trial and Conceptual Domain (With Standard Errors)

\begin{tabular}{|c|c|c|c|c|c|c|c|c|}
\hline \multirow[b]{3}{*}{ Context Trial } & \multicolumn{4}{|c|}{ Animals } & \multicolumn{4}{|c|}{ Nonliving Things } \\
\hline & \multicolumn{2}{|c|}{ RT } & \multicolumn{2}{|c|}{ Errors } & \multicolumn{2}{|c|}{ RT } & \multicolumn{2}{|c|}{ Errors } \\
\hline & $M$ & $S E$ & $M$ & $\overline{S E}$ & $M$ & $S E$ & $M$ & $\overline{S E}$ \\
\hline Same category & $1,083.47$ & 29.28 & 15.00 & 2.16 & $1,102.67$ & 36.48 & 13.33 & 2.64 \\
\hline Different category & $1,088.69$ & 29.08 & 16.67 & 2.92 & $1,112.11$ & 31.22 & 17.22 & 2.88 \\
\hline Switching cost & -5.22 & & -1.67 & & -9.44 & & -3.89 & \\
\hline
\end{tabular}


multiple processing channels, corresponding to a specialization of the semantic system in terms of types of information associated with sensory modalities (e.g., Crutch \& Warrington, 2003; Lauro-Grotto et al., 1997; McCarthy \& Warrington, 1988; Shallice, 1988, 1993).

As was previously mentioned, the results are also consistent with an organization of semantic memory by domainspecific subsystems within modality-specific processing channels. This would explain the larger modality-specific switch costs in Experiment 1 and the absence of significant category switch costs in Experiment 2 that, corresponding to switches between subchannels, could have easily blurred together. This possibility would, however, be inconsistent with what is known about how the brain processes information. For example, fMRI evidence for subregions of the visual-form-processing pathway that are more active for one domain than for another does not imply that those areas are dedicated to processing that domain. In fact, the same regions are active, to some degree, for all domains (e.g., Martin, Wiggs, Ungerleider, \& Haxby, 1996; Moore \& Price, 1999). Moreover, this possibility would also mean that relatively pure domain deficits (e.g., Patient E.W. for animals; reported by Caramazza \& Shelton, 1998) would have to be explained by assuming a selective impairment of that domain subsystem within each major modality-specific channel, which is certainly not a very plausible situation in terms of brain damage. ${ }^{6}$

Another alternative account of the results would be to suppose that the kind of sentences used ("a CONCEPT can be/have FEATURE") favored modality over category switches. This could have happened, since the sentence frame allowed a stronger spreading of activation from feature to modality than from concept to category and/or because features had the greatest impact on brain activation, since they came second in the trial sequence. Thus, one could argue that the two manipulations (maintaining category and manipulating modality or the reverse) are not directly comparable. Although a direct test of this possibility with other kinds of sentences was not made, different evidence would argue against it. In relation to the stronger spreading activation explanation, data from feature production norms seem to disconfirm it. When the stimuli are at the concept level (e.g., Cree \& McRae, 2003, feature norms), many participants give categorical information as features (e.g., is a fruit, is a reptile, etc.). However, when the stimuli are at the feature level (e.g., Underwood \& Richardson, 1956), participants usually select concepts that have those features, and not modalities with which those features can be associated. In relation to the kind of recency as impact explanation, it must be first said that in the language tested, as in many others, this feature after concept structure is that of the usual common sentence, not the reverse. This fact can also lead us to argue that the reverse format could enhance the item (and thereafter the category) just because it is an unusual language format. Moreover, in recent neuroimaging research using the same kind of sentences as those employed here and evaluating brain activation, Simmons, Pecher, Hamann, Zeelenberg, and Barsalou (2003) observed that concept profiles in terms of modality experience (how much of your experience with Item X involves Modality Y) were better predictors of brain activation (also observed to be multimodal) than were feature profiles (how much of your experience with Feature X involves Modality Y). These results seem to show that brain activation in this situation is not influenced by sentence position in a way that favors modality over category switches. Instead, what these results suggest is that the absence of a large category switch may have to do with multimodal activation stemming from the concepts that overlaps, at least partially, between categories. This explanation, however, needs to be further evaluated. In more general terms, evidence from neuroimaging studies has been presented either as not conclusive (e.g., Devlin et al., 2002; Price \& Friston, 2002) or as supporting a multiple semantics account in terms of sensory modalities (Barsalou, Simmons, Barbey, \& Wilson, 2003; Thompson-Schill, 2003), but not in terms of the verbal-visual distinction (Bright, Moss, \& Tyler, 2004; Thompson-Schill, 2003). Nevertheless, as different authors have emphasized (e.g., Caramazza \& Mahon, 2003; Devlin et al., 2002), there are still significant differences in task, materials, and techniques between studies that do not allow for a more definitive conclusion about a modality-specific organization in the semantic system.

Finally, although the present results seem to show that a view of semantic memory organization that does not contemplate a specialization in terms of sensory modalities is probably wrong, they do not exclude the possibility that some part of the information may correspond to an amodal store. Plaut (2002) recently presented a model in which these two possibilities are articulated and provided support for the model in terms of a computational simulation that could account for the pattern of performance observed in optic aphasias. In this model, specialization is influenced by input modality, by the nature of the information, and by a topographic bias favoring short connections. The result would be that regions equidistant from multiple modalities would learn to function in a relatively amodal way, whereas regions near a particular modality would serve functions that are more modality specific. One possibility for testing this hypothesis with the present paradigm would be to use features whose acquisition and representation do not seem dependent (or seem equally dependent) on the multiple modalities evaluated. The main difficulty may be to identify these features. In the initial rating task in Experiment 1, the items that had a nondominant modality of acquisition mainly indicated a similar dominance of touch and vision. However, this may just be a conceptual extension of the crossmodal links of stimulus evaluation found at the perceptual level (e.g., Sathian \& Zangaladze, 2002).

Whereas the possibility of an account of multiple semantics in terms of a combination of modality-specific and amodal systems cannot be excluded on the basis of the present results, a defense of an account in which separate systems of amodal symbols represent the information from each modality does not seem tenable. In the first place, evidence that sensory variables influence concep- 
tual processing (e.g., Stanfield \& Zwaan, 2001; Zwaan, Stanfield, \& Yaxley, 2002) is contrary to the idea that concepts are represented by amodal symbols. In the second place, even if this were to be true, it would undermine the associated idea of a single amodal system of semantic knowledge.

In conclusion, there is growing evidence from cognitive psychology, neuropsychology, and neuroimaging for a specialization of semantic organization in terms of sensory modalities. This evidence should now be integrated into more well defined theoretical proposals that can be evaluated at these different levels.

\section{REFERENCES}

Barsalou, L. W., Simmons, W. K., Barbey, A. K., \& Wilson, C. D. (2003). Grounding conceptual knowledge in modality-specific systems. Trends in Cognitive Sciences, 7, 84-91.

Bright, P., Moss, H., \& Tyler, L. K. (2004). Unitary vs multiple semantics: PET studies of word and picture processing. Brain \& Language, 89, 417-432.

Capitani, E., Laiacona, M., Mahon, B., \& Caramazza, A. (2003). What are the facts of semantic category-specific deficits? A critical review of the clinical evidence. Cognitive Neuropsychology, 20, 213-261.

Caramazza, A., Hillis, A. E., Rapp, B. C., \& Romani, C. (1990). The multiple semantics hypothesis: Multiple confusions? Cognitive Neuropsychology, 7, 161-189.

Caramazza, A., \& Mahon, B. Z. (2003). The organization of conceptual knowledge: Evidence from category-specific semantic deficits. Trends in Cognitive Sciences, 7, 354-361.

Caramazza, A., \& Shelton, J. R. (1998). Domain-specific knowledge systems in the brain: The animate-inanimate distinction. Journal of Cognitive Neuroscience, 10, 1-34.

Chang, T. M. (1986). Semantic memory: Facts and models. Psychological Bulletin, 99, 199-220.

CoHen, J. (1988). Statistical power analysis for the behavioral sciences (2nd ed.). Hillsdale, NJ: Erlbaum.

Cree, G. S., \& McRae, K. (2003). Analyzing the factors underlying the structure and computation of the meaning of chipmunk, cherry, chisel, cheese, and cello (and many other such concrete nouns). Journal of Experimental Psychology: General, 132, 163-201.

Crutch, S. J., \& Warrington, E. K. (2003). The selective impairment of fruit and vegetable knowledge: A multiple processing channels account of fine-grain category specificity. Cognitive Neuropsychology, 20, 355-372.

Devlin, J. T., Russell, R. P., Davis, M. H., Price, C. J., Moss, H. E., FADILI, M. J., \& TYLER, L. K. (2002). Is there an anatomical basis for category-specificity? Semantic memory studies in PET and fMRI. Neuropsychologia, 40, 54-75.

Farah, M. J., \& McClelland, J. L. (1991). A computational model of semantic memory impairment: Modality specificity and emergent category specificity. Journal of Experimental Psychology: General, 120, 339-357.

Forde, E. M. E., \& Humphreys, G. W. (1999). Category-specific recognition impairments: A review of important case studies and influential theories. Aphasiology, 13, 169-193.

Kavcic, V., KraR, F. J., \& Doty, R. W. (1999). Temporal cost of switching between kinds of visual stimuli in a memory task. Cognitive Brain Research, 9, 199-203.

Lauro-Grotto, R., Piccini, C., \& Shallice, T. (1997). Modalityspecific operations in semantic dementia. Cortex, 33, 593-622.

Los, S. A. (1999). Identifying stimuli of different perceptual categories in mixed blocks of trials: Evidence for cost in switching between computational processes. Journal of Experimental Psychology: Human Perception \& Performance, 25, 3-23.

Marques, J. F. (1997). Medidas de tipicidade e familiaridade para diferentes categorias de itens verbais [Typicality and familiarity measures for different categories of verbal items]. Revista Portuguesa de Psicologia, 32, 35-55.

Marques, J. F. (2002). Normas de associação para 303 palavras portuguesas [Associative norms for 303 Portuguese words]. Revista Portuguesa de Psicologia, 36, 35-43.

Martin, A., Wiggs, C. L., Ungerleider, L. G., \& Haxby, J. V. (1996). Neural correlates of category-specific knowledge. Nature, 379, 649652 .

McCARThy, R. A., \& WARRINGTON, E. K. (1988). Evidence for modalityspecific meaning systems in the brain. Nature, 334, 428-430.

Moore, C. J., \& Price, C. J. (1999). A functional neuroimaging study of the variables that generate category-specific object processing differences. Brain, 122, 943-962.

Moss, H. E., Tyler, L. K., \& Devlin, J. (2002). The emergence of category-specific deficits in a distributed semantic system. In E. M. E. Forde \& G. W. Humphreys (Eds.), Category-specificity in brain and mind (pp. 115-148). Hove, U.K.: Psychology Press.

PAIVIO, A. (1971). Imagery and verbal processes. New York: Holt.

Pecher, D., Zeelenberg, R., \& Barsalou, L. W. (2003). Verifying conceptual properties in different modalities produces switching costs. Psychological Science, 14, 119-124.

Plaut, D. C. (2002). Graded modality-specific specialization in semantics: A computational account of optic aphasia. Cognitive Neuropsychology, 19, 603-639.

Price, C. J., \& Friston, K. J. (2002). Functional imaging studies of neuropsychological patients: Applications and limitations. Neurocase, 8, 345-354.

Raaijmakers, J. G. W., Schrijnemakers, J. M. C., \& Gremmen, F. (1999). How to deal with "the language-as-fixed-effect fallacy": Common misconceptions and alternative solutions. Journal of Memory \& Language, 41, 416-426.

Sathian, K., \& Zangaladze, A. (2002). Feeling with the mind's eye: Contribution of visual cortex to tactile perception. Behavioural Brain Research, 135, 127-132.

Shallice, T. (1988). Specialization within the semantic system. Cognitive Neuropsychology, 5, 133-142.

Shallice, T. (1993). Multiple semantics: Whose confusions? Cognitive Neuropsychology, 10, 251-261.

Simmons, W. K., Pecher, D., Hamann, S. B., Zeelenberg, R., \& BarSALOU, L. W. (2003, March). fMRI evidence for modality-specific processing of conceptual knowledge on six modalities. Poster presented at the 10th Annual Meeting of the Cognitive Neuroscience Society, New York.

Spence, C., Nicholls, M. E. R., \& Driver, J. (2001). The cost of expecting events in the wrong sensory modality. Perception \& Psychophysics, 63, 330-336.

Spence, C., Pavani, F., \& Driver, J. (2000). Crossmodal links between vision and touch in covert endogenous spatial attention. Journal of Experimental Psychology: Human Perception \& Performance, 26, 1298-1319.

StANFIELD, R. A., \& ZwAan, R. A. (2001). The effect of implied orientation derived from verbal context on picture recognition. Psychological Science, 12, 153-156.

Thompson-SCHILL, S. L. (2003). Neuroimaging studies of semantic memory: Inferring "how" from "where." Neuropsychologia, 41, 280-292.

Tranel, D., Logan, C. G., Frank, R. J., \& Damasio, A. R. (1997). Explaining category-related effects in the retrieval of conceptual and lexical knowledge for concrete entities: Operationalization and analysis of factors. Neuropsychologia, 35, 1329-1339.

Tyler, L. K., Moss, H. E., Durrant-Peatfield, M. R., \& Levy, J. P. (2000). Conceptual structure and the structure of concepts: A distributed account of category-specific deficits. Brain \& Language, 75 , 195-231.

UnderWood, B. J., \& RichaRdson, J. (1956). Some verbal materials for the study of concept formation. Psychological Bulletin, 53, 84-95.

Warrington, E. K., \& Shallice, T. (1984). Category-specific impairment. Brain, 107, 829-853.

Zwaan, R. A., Stanfield, R. A., \& Yaxley, R. H. (2002). Language comprehenders mentally represent the shape of objects. Psychological Science, 13, 168-171. 


\section{NOTES}

1. In accordance with the more recent consideration of categoryspecific deficits (e.g., Caramazza \& Mahon, 2003; Cree \& McRae, 2003), domain is used to refer to the tripartite distinction of animals, fruits/vegetables, and nonliving things, whereas category is used to further distinctions within domains (e.g., vehicles vs. tools; birds vs. insects).

2. The verbal-visual distinction (e.g., Paivio, 1971) does not really correspond to different sensory modalities but, rather, reflects a combination of content, context, and format differences (Bright, Moss, \& Tyler, 2004; Plaut, 2002).

3. As will be apparent from the description of method, the number of trials needed for the manipulation of one variable was so large (especially in terms of fillers) that we preferred to use two experiments with a one-independent-variable design, instead of only one experiment with a two-variable (i.e., modality and category) design.

4. Plant life (mainly fruits and vegetables), the third conceptual domain generally considered (Caramazza \& Mahon, 2003; Cree \& McRae, 2003), was not tested, because there were not enough familiar concepts in this domain to allow the manipulations needed.

5. Inasmuch as the same critical target trials were used for the two switch conditions through a counterbalanced design, item analyses were not performed, since in this situation the traditional $F_{1}$ is the correct test statistic (Raaijmakers, Schrijnemakers, \& Gremmen, 1999).

6 . I thank one anonymous reviewer for raising this point.

\begin{tabular}{|c|c|c|c|}
\hline \multicolumn{4}{|c|}{$\begin{array}{c}\text { APPENDIX } \\
\text { Target and Context Concept-Feature Pairs (English Equivalents) } \\
\text { Used in Experiments } 1 \text { and } 2\end{array}$} \\
\hline \multicolumn{2}{|c|}{ Target Trials } & \multicolumn{2}{|c|}{ Context Trials } \\
\hline Living & Nonli & Living & Nonliving \\
\hline \multicolumn{4}{|c|}{ Experiment 1} \\
\hline $\begin{array}{l}\text { Dog-bark } \\
\text { Duck-quack } \\
\text { Tiger-growl } \\
\text { Cat-silky } \\
\text { Worm-flaccid } \\
\text { Canary-yellow } \\
\text { Kangaroo-beige } \\
\text { Zebra-stripes } \\
\text { Owl-hoot } \\
\text { Monkey-screech } \\
\text { Parrot-talk } \\
\text { Rabbit-soft } \\
\text { Snail-viscous } \\
\text { Panther-black } \\
\text { Peacock-colorful } \\
\text { Bear-dark }\end{array}$ & $\begin{array}{l}\text { Revolver-bang } \\
\text { Telephone-ring } \\
\text { Wardrobe-creak } \\
\text { Skirt-rough } \\
\text { Blouse-satin } \\
\text { Lamp-light } \\
\text { Saw-flash } \\
\text { Screw-brass } \\
\text { Machine gun-noisy } \\
\text { Bell-resonate } \\
\text { Bed-squeak } \\
\text { Sweater-cottony } \\
\text { Scarf-smooth } \\
\text { Fridge-white } \\
\text { Knife-silvery } \\
\text { Scissors-shiny }\end{array}$ & $\begin{array}{l}\text { Bee-buzz } \\
\text { Lion-roar } \\
\text { Cow-moo } \\
\text { Octopus-slippery } \\
\text { Lobster-rough } \\
\text { Mouse-gray } \\
\text { Giraffe--spots } \\
\text { Camel-cream } \\
\text { Horse-pinto } \\
\text { Ladybug-markings } \\
\text { Grasshopper-green } \\
\text { Pig-grunt } \\
\text { Deer-brown } \\
\text { Donkey-heehaw } \\
\text { Turtle-hard } \\
\text { Chicken-cluck }\end{array}$ & $\begin{array}{l}\text { Whip-lash } \\
\text { Clock-tick-tock } \\
\text { Radio-loud } \\
\text { Waistcoat-velvety } \\
\text { Hat-warm } \\
\text { TV-colors } \\
\text { Ruler-transparent } \\
\text { Brick-red } \\
\text { Cannon-wheels } \\
\text { Mirror-reflect } \\
\text { Sofa-fluffy } \\
\text { Shoe-black } \\
\text { Cap-blue } \\
\text { Mattress-damp } \\
\text { Hammer-hard } \\
\text { Rubber-elastic }\end{array}$ \\
\hline \multicolumn{4}{|c|}{ Experiment 2} \\
\hline $\begin{array}{l}\text { Chicken-cluck } \\
\text { Duck-quack } \\
\text { Mouse-gray } \\
\text { Giraffe-spots } \\
\text { Worm-flaccid } \\
\text { Pig-grunt } \\
\text { Dog-bark } \\
\text { Peacock-colorful } \\
\text { Camel-cream } \\
\text { Turtle-hard }\end{array}$ & $\begin{array}{l}\text { Revolver-bang } \\
\text { Telephone-ring } \\
\text { Lamp-light } \\
\text { Scissors-shiny } \\
\text { Skirt-rough } \\
\text { Machine gun-noisy } \\
\text { Door-creak } \\
\text { Chair-blue } \\
\text { Screw-brass } \\
\text { Blouse-satin }\end{array}$ & $\begin{array}{l}\text { Bee-buzz } \\
\text { Lion-roar } \\
\text { Zebra-stripes } \\
\text { Canary-yellow } \\
\text { Octopus-slippery } \\
\text { Parrot-talk } \\
\text { Donkey-heehaw } \\
\text { Deer-brown } \\
\text { Ladybug-markings } \\
\text { Bear-furry }\end{array}$ & $\begin{array}{l}\text { Radio-loud } \\
\text { TV-low } \\
\text { Shoe-dark } \\
\text { Brick-red } \\
\text { Sweater-cottony } \\
\text { Whip-lash } \\
\text { Clock-tick-tock } \\
\text { Mirror-reflect } \\
\text { Pliers-black } \\
\text { Scarf-smooth }\end{array}$ \\
\hline
\end{tabular}

(Manuscript received July 27, 2004;

revision accepted for publication December 12, 2004.) 